\title{
Endoscopic resection of the submandibular gland: A new surgical approach.
}

Sumeet Karn, Archit Pandit

B and B Hospital, lalitpur

\begin{abstract}
Objectives/Hypothesis: Submandibular gland excision is traditionally performed by the transcervical approach. To avoid or reduce visible scarring and nerve injury, diverse innovative surgical trials have been reported. Here we report a patient who had an endoscopic submandibular gland resection via a hairline incision.

Methods: A 36-year-old woman presented with a right submandibular gland tumor that was found on a routine check-up. The submandibular gland was resected under endoscopic assistance via a posterior hairline incision using an ultrasonic scalpel.
\end{abstract}

Results: The resection was successful, causing no acute complications, such as neural injury, hematoma, or seroma formation. The incision scar healed with an excellent cosmetic result.

Conclusions: Endoscopic submandibular gland resection via a hairline incision was feasible and resulted in an excellent surgical and cosmetic outcome. 